\title{
Adopting self-care interventions for sexual and reproductive health in the Eastern Mediterranean Region ${ }^{1}$
}

Citation: Adopting self-care interventions for sexual and reproductive health in the Eastern Mediterranean Region. East Mediterr Health J. 2019;25(6):441-442 https://doi.org/10.26719/2019.25.6.441

Copyright (C) World Health Organization (WHO) 2019. Some rights reserved. This work is available under the CC BY-NC-SA 3.o IGO license (https:// creativecommons.org/licenses/by-nc-sa/3.o/igo).

\section{Introduction}

Self-care interventions for sexual and reproductive health remain crucial to ensure continuity and linkages of health care services between primary health care services and communities. Initiating self-care for sexual and reproductive health and rights at policy/regulation, programmatic and service delivery levels will build a strong platform for adaptation of the WHO consolidated guideline on self-care interventions for sexual and reproductive health and rights (1).

In order to develop this, a consultative meeting on adopting self-care interventions for sexual and reproductive health in the Eastern Mediterranean Region was held in Beirut, Lebanon, from 16 to 18 April 2019. The meeting was organized by the WHO Regional Office for the Eastern Mediterranean, Cairo, Egypt, and WHO headquarters, Geneva, Switzerland (2).

The objectives of the meeting were to:

- update the processes and timelines of development of the WHO guideline on self-care interventions for sexual and reproductive health and rights;

- examine the current situation of self-care interventions for sexual and reproductive health in participating countries;

- determine the main factors and underlying causes facilitating and preventing adoption of self-care interventions at the country level, and suggest actions to address these; and

- outline a standardized approach and methodology of work to adapt the WHO guideline's evidence-based interventions in the Eastern Mediterranean Region.

The meeting was attended by 30 participants from five countries (Egypt, Islamic Republic of Iran, Lebanon, Morocco and Syrian Arab Republic), in addition to representatives from the United Nations Population Fund (UNFPA), United Nations High Commission for Refugees (UNHCR), International Planned Parenthood Federation (IPPF) and WHO.

\section{Summary of discussions}

The WHO consolidated guideline on self-care interventions for sexual and reproductive health and rights (1) was developed with political and cultural sensitivities as key considerations. The guideline took into account humanitarian and conflict settings which represent a critical challenge. Self-care has great potential to deliver health care in post-conflict settings, particularly when implementation is tailored to overcome existing obstacles. The guideline is designed to be interactive, so that whenever new evidence is generated, revision of the guideline will be considered.

Five key new topics were discussed for inclusion in the recommendations of the consolidated guideline:

- self-administered injectable contraception, or depomedroxyprogesterone acetate (DMPA), should be made available as an additional approach to deliver injectable contraception for individuals of reproductive age;

- over-the-counter oral contraceptive pills (OCPs) should be made available without a prescription for individuals using OCPs;

- human papillomavirus self-sampling should be made available as an additional approach to sampling in cervical cancer screening services for individuals aged $30-60$ years;

- home-based ovulation predictor kits (OPKs) should be made available as an additional approach to fertility management for individuals attempting to become pregnant; and

- self-collection of samples for Neisseria gonorrhoeae and Chlamydia trachomatis should be made available as an additional approach to deliver STI testing services; and self-collection of samples for Treponema pallidum (syphilis) and Trichomonas vaginalis may be considered as an additional approach to deliver STI testing services.

Research and self-care interventions for sexual and reproductive health was debated through a panel discussion with emphasis on prioritization of research areas, focus on country specific context and research documentation process at the country level. It was noted that there is an urgent need for further research using qualitative studies targeting women's and communities' real needs, focusing on the types of information required, identifying self-care interventions already adopted, and

This report is extracted from the Summary report on the Informal consultative meeting on adopting self-care interventions for sexual and reproductive health in the Eastern Mediterranean Region, Beirut, Lebanon, 16-18 April 2019 (http://applications.emro.who.int/docs/IC_Meet_Rep_2019_ EN_23553.pdf?ua=1). 
analysing their relationships with their health providers at primary health care level. Other subjects to be explored for research included people's perceptions of self-care, the perspective of the health workforce on self-care at different levels and in different settings, and the main barriers to implementing self-care.

\section{Recommendations}

\section{To Member States}

- Initiating self-care for sexual and reproductive health at policy and regulation, programmatic and service delivery levels.

- Adopting self-care for sexual and reproductive health interventions within a continuum of care approach.

- Strengthening existing sexual and reproductive health programmes through adoption of self-care interventions.

- Considering the five self-care sexual and reproductive health interventions as recommended by WHO to be published in the consolidated guideline on self-care interventions for sexual and reproductive health and rights in 2019.

- Considering context specificities to adapt and adopt sexual and reproductive health self-care interventions.

- Ensuring linkages, integration and multisectoral approaches during the adaptation phase of self-care interventions at country level.

- Considering monitoring, evaluation, documentation and research to assess the impact of self-care interventions and adjust the care according to women's and communities needs and priorities.

\section{To WHO}

- Finalizing and publishing the WHO consolidated guideline on self-care interventions for sexual and reproductive health and rights during August 2019, and translating into Arabic by September 2019.

\section{References}

1. World Health Organization. WHO consolidated guideline on self-care interventions for health: sexual and reproductive health and rights. Geneva: World Health Organization; 2019 (https://www.who.int/reproductivehealth/publications/self-care-interventions/en/).

2. Informal consultative meeting on adopting self-care interventions for sexual and reproductive health in the Eastern Mediterranean Region, Beirut, Lebanon, 16-18 April 2019 (http://applications.emro.who.int/docs/IC_Meet_Rep_2019_EN_23553.pdf?ua=1). 